\title{
PיL- -195.7
}

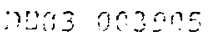

DISSIPATIVE EFFECTS ON FINITE-LARMOR-RADIUS MODIFIED

MAGNETOHYDRODYNAMIC BALLOONING MODES

J.W. Connor, " Liu Chen, and M.S. Chance

Plasma Physics Laboratory, Princeton University

Princeton, New Jersey 08544

\section{ARSTRACT}

Finite-ton-Larmor-radius (FLR) effects provide a hand of additional stability for values of $\beta(\equiv$ plasala pressure/magnet1c pressure) exceeding tale 1 imft predtcted for ideal magnetohydrodynamic (MHD) ballooning modes. Wt examine the effect of particle collisions on the stable modes of the FLI modifte 1deal theory that exist in this range of $\beta$ values.

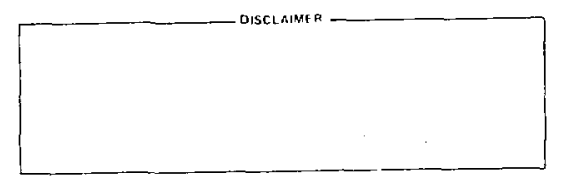

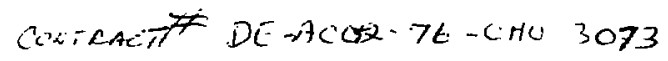

\footnotetext{
*Permanent addres:: Culham Laboratory, Ablingdon, Oxon OX143DB, U.K.
} (Euratom,UKAEA Fusion As sociation) 


\section{INTRDDUCT $10 N$}

The theory of high-n ideal MHD ballooning rodes in an axisymmetric torus predicts that, for given pressure and current proffles, there will be a critical value of $\beta, 1 . e . \beta_{c}{ }^{\text {MHD }}$, that will cause instability $[1,2,3]$. When $\beta>\beta_{C}^{M H D}$ there will be discrete modes with frequencles

$$
\omega_{M H D}=\omega_{A}\left(\beta / \beta_{C} M H D-1\right)^{1 / 2} \text {, }
$$

where $\omega_{A}$ is the Alfven frequency $V_{A} / L_{C}$ with $V_{A}$ the Alfven speed and $L_{\text {. }}$ the connection length. Below this value of $\beta$ lies a continum of stable odes with $\underset{\mathrm{MHD}}{2}>0$. In the simplest model $[4,5]$ the effect of finite lon $L$ mor radius is to cause the substitution $\omega^{2} \rightarrow \omega\left(\omega-\omega_{\star_{1}}\right)$ for the cal eigenvalue $\omega^{2}$ of IdeaI MHD, where $\omega_{\star_{1}}=k_{1} a_{1} V_{T 1} / r_{n}$ is the fon diamagnetic drift frequency. Here $k_{1}$ is the wave number perpendicular to the magnetic ficld, $a_{1}$ is the ion Larmor radius, $v_{T j}$ is the thermal speed of specles $j$ and $r_{n}$ is tine density scale length. Equivalently, $\omega_{\star_{1}}-\sqrt{b_{1} \beta} \omega_{A} L_{C} / r_{n}$ where $b_{1}=k_{1}{ }^{2} a_{1}{ }^{2}$. Thus $1 t$ is clear that $w$ is now given by

$$
\omega=\frac{\omega_{1}}{2} \pm\left(\omega_{\star_{1}}^{2} / 4+\omega_{M H D}^{2}\right)^{1 / 2}
$$

and this corresponds to an increase in the critical $\beta$ for instability to

$$
\beta_{c}^{F L R}=\beta_{c}^{M H D}\left(1+\frac{b_{1}}{4 E}\right),
$$

where $\varepsilon \equiv r_{n} / L$ is the inverse aspect $r a t 10, \beta_{c}^{M H D}=\varepsilon$ and $w$ is approximately given by 


$$
\omega=\frac{\omega_{\star_{1}}}{2} \pm \omega_{\mathrm{A}}\left(\beta / \beta_{\mathrm{C}}{ }^{\mathrm{FLR}}-1\right)^{1 / 2} .
$$

When $\beta>\beta_{c}^{\text {FLK }}$ there 1 s one stable and one unstable mode which connect to two discrete stable modes when $\beta<\beta_{c}$ FLR . As $\beta$ is further reduced to $\beta_{c}$ MHD, the real parts of the frequencles of these rodes approach zero and $\omega_{\star 1}$ respectively. Finally, when $\beta<\beta_{C}^{M H D}$, these discrete stable modes are replaced by a stable continum, since then $w\left(\omega-\omega_{*_{1}}\right)>0$ (c.f. $\omega_{\mathrm{MHD}}^{2}>0$ in the ideal case [l].

Now in the absence of FLR effects $t$ is known that unstable high n resistive balloontng modes are present for $\beta<\beta_{c}^{M H D}[6]$. It Is the purpose of this note to examline the effect of reststlvity and perpendicular ton viscosfty on the stable discrete modes present in the range $\beta_{C}{ }^{M H D}<\beta<\beta_{C} F L R$.

In Sec. I I we derlve the required elgenvalue equation from a kinetic description of tons and electrons in the approprtate collislonal regime. For the electrons we solve the klnetic equation in the weakly colilsional fluid IImit $v_{e}>w$, but $w v_{e} \sim k_{\|}{ }^{2} v_{T e}{ }^{2}$, where $v_{j}$ is the colliston freyuency, $v_{T j}$ the thermal velocity of specles $j$ and $k_{\|}$is the wave number parallel to the Inagnetic fleld. For the lons we constder $k_{1}$ suffictently large so that $\omega \sim \omega_{\star}>k_{\|} V_{T 1}$ and, to include lon viscosity, $\omega \sim v_{1} b_{1}$. Substitution of the electron and ton responses in the quasi-neutrality and Maxwell's equations provides an elgenvalue equation. Solutions of this equation (both qualitative and numerlcaj) for a large aspect-ratio equilibrium with circular surfaces are discussed in Sec. III. 
II. THE EQUATIONS FOR RESISTIVE MODES

In this section we Introduce the equilibrium, the ballooning representation, the gyro-kinetic equation, from which we derive useful moment equations, and Maxwe 11 's equations.

A. Equilibrium.

We adopt the axisymmetric orthogonal flux coordinates $\psi, \zeta, \chi$ witii Jacobian $J$, wher: $\psi$ is the pololdal flux within a magnetic surface, $\zeta$ the toroidal angle, and $\chi$ a pololdal angle varlable. In these coordinates the magnetic field is expressible as [1]

$$
\underline{B}=-\nabla \psi \times \underset{\sim}{\nabla} \zeta+I(\Psi) \underset{\sim}{\nabla},
$$

where $I(\psi)$ is the prescribed toroldal field function, and the gradient operator may be written as

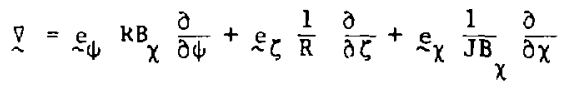

with $R$ being the major radius. The safety factor then becomes

$$
q=\frac{1}{2 \pi} \oint v d x
$$

with $v=I J / R^{2}$

B. Ballooning Representation.

Perturbed quantities are represented In the form [2] 


$$
\phi(\psi, \zeta, \chi,)=\sum_{p=-\infty}^{\infty} \bar{\phi}(\psi, \zeta, \chi-2 \pi p)
$$

which is automatically perforic in $\chi$. Therefore, for high-n perturbations, one can use an elkonal form to represent modes with short wavelength perpendicular to the magnetic field but long wavelength along 1 , without a conflict with perlodicity in the presence of shear. Thus we write

$$
\bar{\phi}=\hat{\phi}(\downarrow, x) \exp (1 \mathrm{nS})
$$

with

$$
S=\zeta-\int_{v} v d x+\int^{\psi} k(\psi) d \psi
$$

so that $n \cdot \nabla S=0$ where $n=B / B . \hat{\phi}$ is a slowly varylng function of $\psi$ and $\chi$ which satisfles the same equation as $\phi$ but is no longer a perlodtc function of $\chi$ and must he suitahly well behaved as $|\chi| \rightarrow \infty$. We shall

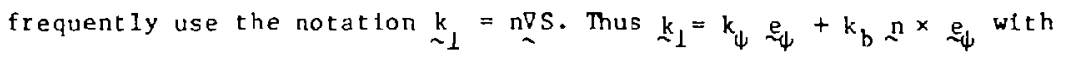

$$
\left.k_{\psi}=-\mathrm{nRB}_{\chi}[\}^{\chi} \mathrm{d} \chi \frac{\partial \nu}{\partial \psi}-\mathrm{k}(\psi)\right]
$$

and

$$
k_{b}={ }_{n} B / R B
$$

C. Grokinetic Equation.

As a result of the small ratio of their Larmor radil to mascroscopic scales the plasma lons and electrons of a Maxwellian plasma have perturbed distribution functions of the form [s] 


$$
\hat{\mathrm{f}}=\frac{-e \hat{\phi}}{\mathrm{T}} \mathrm{F}_{M}+\hat{\mathrm{h}}(\varepsilon, \mu, \chi) \exp (1 \mathrm{~L})
$$

where $\phi$ is the electrostatic potential, $F_{M}$ is a Maxwellian,

$$
F_{M}=n_{0}\left(\frac{m}{2 \pi T}\right)^{3 / 2} \exp \left(\frac{-\pi E}{T}\right),
$$

$\varepsilon=v^{2} / 2$ is the energy and $\mu=v_{1}^{2} / 2 B$ is the magnetic moment per unit mass, $\eta$ is the gyro-phase angle so that $\left.\underset{\sim}{\mathrm{v}}=v_{\| \sim} \mathrm{n}+v_{\perp} \cos \eta \varepsilon_{\psi}+\operatorname{sim} n \times \xi_{\psi}\right)$,

$$
\mathrm{d}^{3} \mathrm{v}=\mathrm{Bd \mu} \mathrm{d} \varepsilon \operatorname{dn} /\left|\mathrm{v}_{\mathrm{y}}\right|,
$$

and

$$
L=\left(v_{1} / 8\right)\left(k_{\psi} s 1 m-k_{b} \cos \eta\right)
$$

with $\&$ being the gyrofrequency.

The function $\hat{h}$ is a solution of the gyro-kinetic equation $[5, i$

$$
\begin{aligned}
V_{G \perp} D \cdot \nabla \hat{h} & -1 \hat{h}\left(\omega-\omega_{D}\right)-\left\langle e^{-1 L} c\left(\hat{h e} e^{1 L}\right)\right\rangle_{\eta} \\
& =\frac{-1 e}{T} F_{M}\left(\omega-\omega_{\star}^{T}\right)\left[J_{0}(\alpha)\left(\hat{\phi}-\frac{V_{1}}{c} \hat{A}_{\|}\right)+J_{1}(\alpha) \frac{V_{1}}{k_{1} c} \delta \hat{B}_{\|}\right],
\end{aligned}
$$

where $\mathrm{C}$ is the usual Fokker-Planck collisional operator, \langle\rangle$_{\eta}$ represents a gyrophase average, and

$$
\omega_{D}=\frac{q_{B} B}{T} \omega_{B}+\frac{m v_{H}^{2}}{T} \omega_{K}
$$


with $\omega_{B}=(T / m Q B) \underline{n} \times \nabla B \cdot k_{1}$ and $\omega_{x}=(T / m Q B) \underline{n \times}(n \bullet \nabla n) \cdot k_{1} \cdot J_{0}$ and $J_{1}$ are Bessel functions of argiment $\alpha=k_{1} v_{1} / \Omega, \omega *^{T}=\omega *[1+\eta(m e / T-3 / 2)]$ with $\omega_{*}=(\mathrm{ncT} / \mathrm{e}) \mathrm{d}\left(\mathrm{In} \mathrm{n}_{0}\right) / \mathrm{d} \psi$ and $\eta=\mathrm{d}(\operatorname{lnT}) / \mathrm{d}\left(\ln \mathrm{n}_{0}\right)$. $\hat{A}_{\|}$and $\delta \hat{B}_{\|}$are the components of the vector potential and the perturbed magnetic fleld along the equilibrim fleld. Here we use the Coulomb gauge div $\underset{A}{\hat{A}}=0$.

We emphasize that this equation contalns secular terms in $x$ througli $\omega_{D}$ and $\alpha$ artsing from shear and that we seek non-pertodic solutions defined an the interval $-\infty<\chi<\infty$ which are sultably well behaved at infinity. In this work we shall cons!der the long wavelengths 11 m1t $b<<1$ where $b=k_{1}^{2} I / 0 \Omega \Omega^{2}$ so that in this equation the collision term and Bessel functions can be expanded $1 \pi L$ and $\alpha$.

D. Eiectron Solution.

Using the symbol $k_{\| l}=n \cdot \nabla \phi / \phi$, we solve the electron gyro-kinetic equation tn the limit $\omega / k_{\|} V_{T e}-k_{\|} V_{e} / v e \ll 1, b_{e} \ll 1$. To simplify notation, we now suppress electrcin species suffices.

Thus, in leading order (and writing $\phi$ tor $\hat{\phi}$, etc.), we have

$$
C\left(h_{0}\right)=0 \quad,
$$

which impiles a Maxwellian solution

$$
h_{0}=\frac{n^{-}}{n_{0}} F_{M}
$$

where $n^{\prime}$ is the non-adiabatic density perturbation. For simplicity, we shall ignore equilibrium temperature gradients and the resulting temperature perturbations. 
In next order, we have

$$
C\left(h_{1}\right)=v_{\|} n \cdot \nabla_{0} h_{0}-1\left(\omega-\omega_{*}\right)\left(\frac{e F M}{T c}\right) v_{\|} A_{\|}
$$

and, finally,

$$
C\left(r_{2}\right)=v_{B} n \cdot \nabla h_{1}-1\left(\omega-w_{D}\right) h_{0}+1\left(\omega-w_{\star}\right) \frac{e F_{M}}{T}\left(\phi+\frac{v_{1} 2}{2 \Delta c} \delta B_{\|}\right) .
$$

The parallel current arises from the solutton of Eq. (20) for $\mathrm{h}_{1}$, using Eq. (19) for $h_{0}$. This resembles the problem solved by Spltzer [8] and we find

$$
j_{\|}=\sigma\left[\frac{\mathrm{T}}{\mathrm{e} n_{0}} \mathrm{n} \cdot \nabla n_{\mathrm{e}}^{\prime}+1\left(\omega-w_{\star_{e}}\right) \frac{A_{\|}}{c}\right] \text {, }
$$

where $\sigma$ is the Spitzer conductivity.

E. Ion Solution.

In the Ion case we consider $\omega-\omega_{\star}>k_{\|} V_{T 1}$ (1mposing a lower 11mit on the toroldal mode number $n$ ) and $b_{i}<1$, but $v_{i} b_{i} \sim \omega$, which introduces ton - Ion collistons as a collisional viscosity.

Thus, in leading order, we note that ion - ion colisions dominate the collision term and, hence, obtaln for the lons

$$
C\left(h_{0}\right)=0,
$$

1.e.,

$$
h_{0}=\frac{n^{\prime}}{n_{0}} F_{M}
$$


where ton species' suffices are again suppressed. In next order, we have

$$
\begin{aligned}
-1\left(\omega-\omega_{0}\right) h_{0} & -c\left(h_{1}\right)+\left\langle C\left(h_{0} \frac{L^{2}}{2}\right)\right\rangle_{\eta}-\left\langle L C\left(h_{0} L\right)\right\rangle_{\eta} \\
& =-1\left(\omega \omega_{*}\right) \frac{e F_{M}}{T}\left[\phi-\frac{V_{\|} A_{1}}{c}+\frac{v_{1}^{2}}{22 c} \delta B_{\hbar}\right] .
\end{aligned}
$$

Taking the density morent, we obtaln

$$
\left(\omega-\omega_{K} w_{B}\right) n^{\prime}=\left(\omega-w_{*}\right) \frac{e n_{o}}{T}\left(\phi+\frac{T^{2}}{m \Omega c} \delta B_{\|}\right),
$$

where 1n Eq. (25) density and momentum conservation in ton - ton collisions annthllate the collision term.

F. Paralle1 Current Moment Equation.

We form the moment $\sum e_{j} \int d^{3} V^{1 L}$ of the gyrokinetic equation, Eq.(16), to obtain

$$
\begin{aligned}
& \underline{B} \cdot \underset{\nabla}{\nabla}-\frac{j_{\|}}{B}=1 \omega\left\{\frac{e^{2}}{T} \int d^{3} V F_{M}\left\{\left[1-\left(1-\frac{\omega *}{\omega}\right) J_{0}^{2}(\alpha)\right]_{\phi}-\left(1-\frac{\omega *}{\omega}\right) J_{0}(\alpha) J_{1}(\alpha) \frac{V}{k_{\perp} c} \delta B_{\|}\right\}\right. \\
& -1 \sum e \int d^{3} v_{\omega} D^{h e^{1 L}}+\sum e \int d^{3} v e^{1 L}\left\langle e^{-1 L} c\left(h e^{1 L}\right)\right\rangle_{\eta}+\sum e \int d^{3} V V_{h} h n \cdot \nabla e^{1 L}
\end{aligned}
$$

where we have used Eq. (8) and charge neutrality. If we consider small b, 1.e., Ignoring terms in $b_{e}, b_{1}{ }_{D} / \omega, b_{1}\left(\delta B_{n} / B\right) /(\phi / T)$ (see subsection $G$ ) and $b_{1} k_{\| l} v_{T 1} / \omega_{*}$, we obtain 


$$
\begin{aligned}
& -9- \\
& B \cdot \nabla \frac{j_{\|}}{B}=\frac{1 n_{0} e^{2}}{T_{1}}\left(\omega-w_{\star_{1}}\right) b_{1} \phi+\left(w_{*_{1}}-w_{\star_{e}}\right) \frac{T_{1} \delta B_{\|}}{m_{1} \Omega_{1}} \\
& -\underset{j}{1} e_{j}\left(\omega_{k}+\omega_{B}\right)_{j} \pi_{j}^{\prime}-e \nu_{1} b_{1}^{2} \alpha n_{1}^{\prime}
\end{aligned}
$$

with $\alpha=\frac{3}{10}$ and $v_{1}=4 \pi{ }^{1 / 2} \mathrm{n}_{0} e^{4} \ln \alpha /\left(3 M_{1}{ }^{1 / 2} \mathrm{~T}_{1}{ }^{3 / 2}\right)$.

In derlving this result we have expanded the collision term in $\mathrm{Eq} \cdot$ (27) to order $b_{1}{ }^{2}$, the first non-vanishing contribution, using appropriate conservation properties, and $\mathrm{Eq}$. (25) for the order $b_{1}$ correction to $h_{0}$. The resulting integrals have been evaluated using the projection techniques and expansion procedure of Shkarofsky et a1. [9]. The fon collision term representing the lowest-order collisional non-ambipolar diffusion flux corresponds to lon perpendicular viscosity.

G. Maxwell's Equations.

We can eliminate $\delta B_{\|}$by means of the component of Ampere's law perpendicular to the magnetic surface

$$
I k_{b} \delta B_{\|}=\frac{4 \pi}{c} j_{\psi}
$$

with $j_{\psi}=\int e d^{3} v v_{\perp} \cos h \exp (1 L)$. Equation (29), along with (19) and (24), yields, in the 1 imit $b<<1$,

$$
\delta B_{\|}=4 \pi \sum \frac{e T}{m 8} n^{-}
$$

confirming thai $\delta B_{U} / B \sim \beta \pi / n_{0}$. Using the 1dentity $\left(\beta_{1} / 2\right)\left(\omega_{\star_{1}}-\omega_{\star_{e}}\right)=\left(\omega_{K}-\omega_{B}\right)$ where $\beta_{1}=8 \pi n_{0} T_{1} / B^{2}$, we note that the role of $\delta B_{H}$ is effectively to replace $\omega_{B}$ by $\omega_{K}$ in $E q .(28)$. 
Finally, we have the parallel component of Ampere's law, 1.e.,

$$
k_{\perp}^{2} A_{\| i}=\frac{4 \pi}{c} j_{\|}
$$

H. Ef renvalue Equation.

In the $1 \mathrm{mft} \omega_{\mathrm{D}} / \omega, \beta<1$, Eq. (26) for $n_{1}$ together with quastneutrality implies that

$$
n_{j}^{\prime}=\frac{n_{0} e_{j}}{T_{j}} \phi\left(1-\frac{\omega_{\star} j}{\omega}\right) .
$$

Combining Eqs. (22) and (28) - (32) ylelds the elgenvalue equation

$$
\begin{aligned}
& \stackrel{B \cdot \nabla}{\{}\left\{\frac{\left(k_{1}^{2} / B^{2}\right) B \cdot \nabla \phi}{1+\left[i k_{1}^{2} c / 4 \pi \sigma\left(\omega-\omega_{*_{e}}\right)\right]}\right\}=\frac{-4 \pi n_{0} e^{2} \phi}{T_{1}}\left\{\omega\left(\omega-\omega_{*_{1}}\right) b_{1}+2 \omega_{k i}\left(\omega_{*_{i}} w_{*_{e}}\right)\right. \\
& \left.+\operatorname{tes}\left(\omega w_{\star_{1}}\right) v_{1} b_{1}{ }^{2}\right\}
\end{aligned}
$$

or, employing fluta nolation,

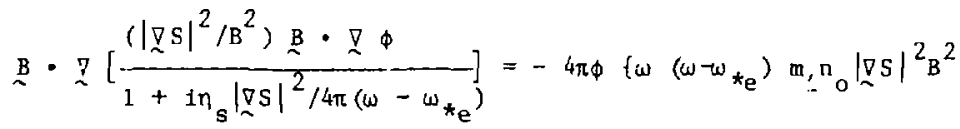

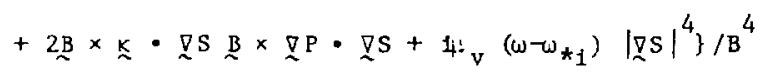

where $p$ is the total pressure, $\eta_{s}$ the spitzer resistivity, $k=\mathfrak{n}$. In and $\mu_{v}=\alpha m_{1}^{2} n_{0} T_{1} \nu_{1} / e^{2}$ is the ion perpendiculatr viscosity. 
III. SOLUTION FOR LARGE ASPECT RATIO TOKAMAK WITH CIRCULAR SURFACES

In this section we consider solutions of Eq. (33) for a large aspect ratio tokamak with circular flux surfaces. In this case the equilibrium can be characterised by two parameters: $\alpha=2 R q{ }^{2} \mathrm{P} / \mathrm{r}_{\mathrm{n}} \mathrm{B}^{2}$ representing the pressure gradient and $s=(r / q) d q / d r$ representing the shear $[10]$. It is conventent to introduce the normalised frequency $\Omega=\omega / \omega_{*_{e}}$ and collision frequency $\nu=$ $v_{\mathrm{e}} \mathrm{r}_{\mathrm{n}} / \mathrm{V}_{\mathrm{Te}}$ where $\sigma=\mathrm{n}_{\mathrm{o}} \mathrm{e}^{2} / \mathrm{mv}$. Equation (33) becomes a differential equation defined on an infinite range of the pololdal angle $\theta$ :

$$
\begin{aligned}
& \frac{d}{d \theta}\left[\frac{\left.1+s^{2} \theta^{2}\right)}{1+1 \nu b_{i 0} 1 / 2 q^{2} / \hat{\beta} E_{n}(\Omega-1)}\right] \frac{d \phi}{d \theta}+2 \hat{\beta}(1+\tau)(\cos \theta+\sin \theta) \\
& +\left(\hat{\beta} b_{10} / \varepsilon_{n} \tau\right) \Omega(\Omega+\tau)\left(1+s^{2} \theta^{2}\right)\left[1+1 \delta_{1} \tau v b_{10} 1 / 2\right. \\
& \left.\times\left(1+s^{2}{ }^{2}\right) / \Omega\right] \phi=0 .
\end{aligned}
$$

He re, $\hat{\beta}=4 \pi q^{2} n_{o} T_{e} / \varepsilon_{n} B^{2}, b_{10}=m_{1} T_{1} n^{2} q^{2} / e^{2} B^{2} r_{n}^{2}, \tau=T_{1} / T_{e}$, and we use the labeling parameter $\delta_{i} 1$ to consider fon-ion collistons independertly of electron collisions. We have solved this equation for $s=q=\tau=1$ and $\varepsilon=b_{10}=0.1$ (consistent with the expansion $\omega_{*}, k_{f} v_{T 1}$ ). With $v=0$ the instability threshold is $\hat{\beta}=0.1997 \equiv \hat{\beta}_{\mathrm{c}}{ }^{\mathrm{F}}$. . We have considered the variation of Im $Q$ with $v$ for the unstable branch when $\hat{\beta}=0.1995$, 1.e., just below $\hat{\beta}_{C}$ FLR, and when $\hat{\beta}=0.1900$, 1ntermediate in the range $\hat{\beta}_{c}^{M H D}<\hat{\beta}<\hat{\beta}_{c}^{F L R}$. The resulte are shriwn in FIg. 1 for two choices of $\delta_{1}$, namely, $\delta_{1}=0$, corresponding to ignoring ion-ion collistons, and $\delta_{i}=1$, characteristic of tinelr being ful ly effective.

It should be noted that the growth rate increases with electron-ion 
collistons. Indeed the behavior can readily be understood qualltatively by perturbation theory. From the general Eq. (34), with $\mu=0$ for simplicity. one can construct schematically a quadratic form

$$
w\left(\omega-w_{\star_{1}}\right)+w_{A}^{2}\left(\frac{\beta}{\beta_{C}^{M M D}}-1+\frac{1 \nu}{\omega-w_{\star_{0}}}\right)=0
$$

where $v^{*}{ }_{a} v$. Thus

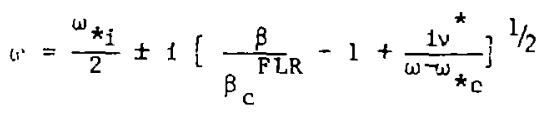

When $\beta \approx \beta_{c}{ }^{\text {FLR }}$, Im $\omega$ has a $v^{1 / 2}$ depentence, wheress when $\beta<\beta_{c}$ FLP. it takes on a linear varlation with $\nu$, features characterlstic of $\mathrm{Fig}, 1$. It can be seen In Fig. I that forrion collisions have a smali stabilizing effect on this mode.

IV. Conclustor.

We have derived an equation to investigate the influence of collisions on high-n ballooning modes in the presence of FLR effects. Calculations have been performed for a large aspect ratio tokamak with clrcular flux surfaces. In the absence of collisional effects there are two modes with real frequencles $1 \mathrm{n}$ the range of $\beta$ value given by

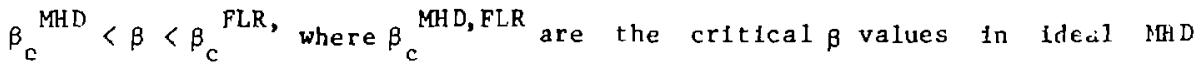
theory and in the presence of FLR, respectively. Electron-ion collisions, 1.e., resistivity, destabilize one of those branches with a growth rate $\gamma \alpha \nu$ when $\beta<\beta_{c}{ }^{\text {FLR }}$, but with $\gamma \alpha \nu^{1 / 2}$ when $\beta \leq \beta_{c}{ }^{\text {FLR }}$. Ion-ion collisions, i.e., fon pe:pendicular viscosity, tend to reduce the se growth rates somewhat. Opposite behaviors are observed for the resistively stable branch. Finally, we remark that FLR effects on resistive vallooning modes in 
the $\beta \leqslant \beta^{M H D}$ regime are current1y under Investigation and will be reported in a future publication.

\section{Acknowl edgment}

This work was supported by Unit:d States Department of Energy Contract No. DEACO2-76-C H03073.

\section{References}

[1]. Connor J. W., Hastie R. J., Taylor J. B., Proc. R. Soc. (London) A365, (1979) 1.

[2]. Glasser A. H., in Proceedings of the Finite $\beta$ Theory horkshop (Varenna, Italy, September 1977), Edited by B. Coppi and W. Sadowski, p.55, National "echnical Infomation Service, Springfieid, Virginia (1979).

[3]. Lee Y. C. Van Dam J. W., $1 \pi$ Proceedings of the Finite $\beta$ Theory Workshop (Varenna, Italy, September 1977), Edited by B. Coppl and $W$. Sadawsk1, p. 93, Naticnal Technical Information Service, Springfield, iirginia (1979).

[4]. Chu M. S. et al. Yhys. Rev. Lett. 41 (1978) 247.

[5]. Tang W. M., Connor J. W., Hastie R. J., Nucl. Fusion 20, (1980) 1439.

[6]. Chance M. S. et al, 1n Plasma Physics and Controlled Naclear Fusion Research (Proc. 7th Int. Conf. Innsbruck, 1978) 1, IAEA, Vlenna, (1979) 677.

[7]. Antonsen T. M., Lane B., Phys. Fluids 23, (I 980) 1205.

[8]. Spitzer Jr. L., Harm R., Phys. Rev. 89 (1953) 977.

[9]. !hkarofsky I. P., Johnston T. W., Bachynskil M. P., The Particle Kinetics of Plasmaj (Addison-Wesley, Reading, Mass., 1966) Chapter 8.

[10]. Connor J. W., Hastle R. J., Taylor J. B., Phys. Rev. Lett. 40, (1978) 396. 


\section{Figure Caption}

Fig. 1. Gowth rate versis electronton collision frequency $v$, for two

different values of $\hat{\beta} \cdot \delta_{1}=1$ and $\delta_{1}=0$ cortespond, respectively, co calculations with and without lon-ion collistons. 


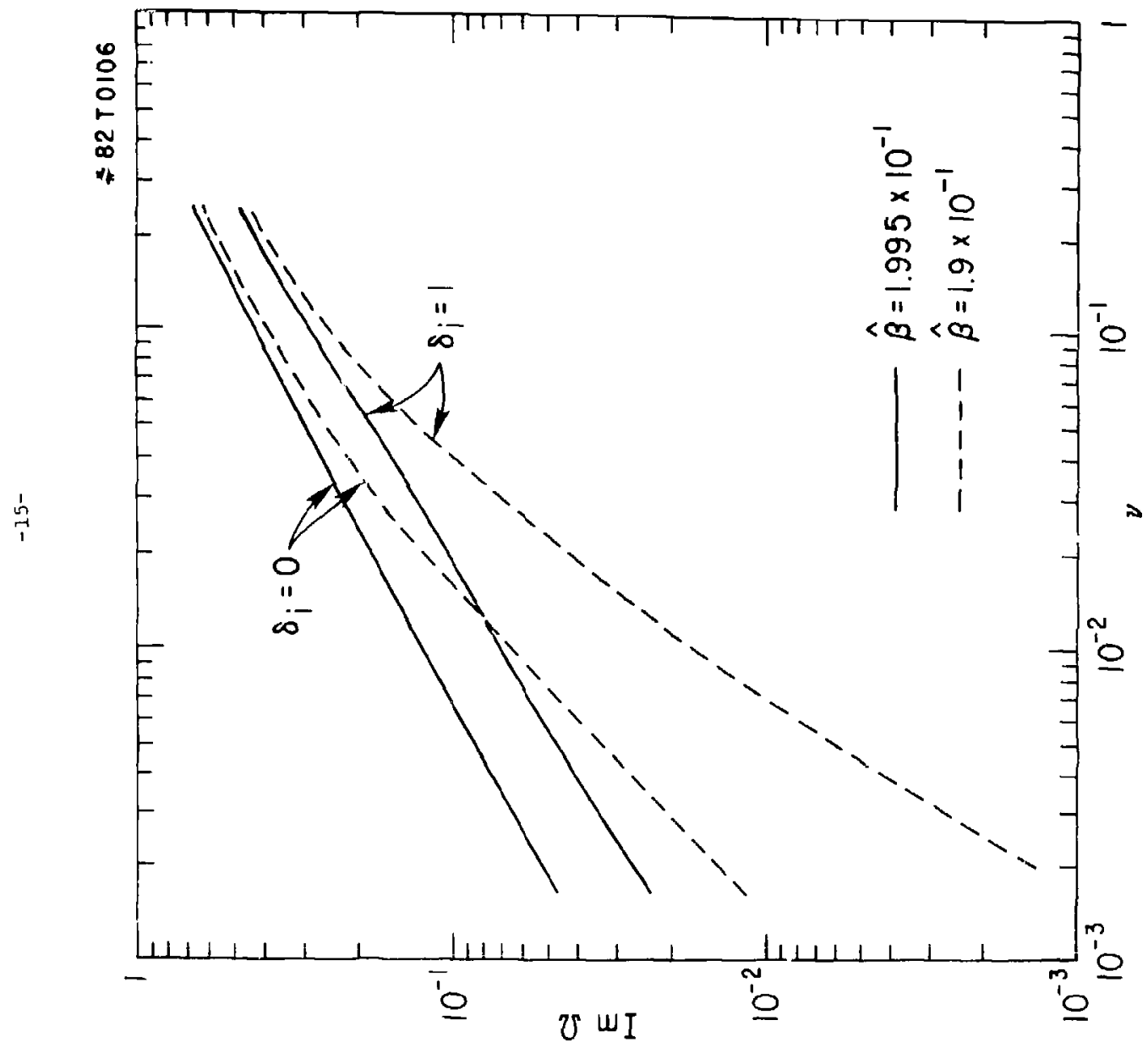

- 